\title{
Study of dielectric coatings for broadband operation of surface-emitting semiconductor lasers
}

\author{
C. Robin Head, ${ }^{1}$ T. Chen Sverre, ${ }^{1}$ Jonathan Woods,,${ }^{1, *}$ Alexander Hein, ${ }^{2}$ Markus Polanik, ${ }^{2}$ \\ Andrew P. Turniull, ${ }^{1}$ Edward A. Shaw, ${ }^{1}$ Peter Unger,${ }^{2}$ Anne C. Tropper, ${ }^{1}$ And \\ VAsilis Apostolopoulos ${ }^{1}$ (1) \\ ${ }^{1}$ School of Physics and Astronomy, University of Southampton, University Road, Southampton, Hampshire SO17 1BJ, UK \\ ${ }^{2}$ Institute of Optoelectronics, Ulm University, Albrecht-Einstein-Allee 45, 89081 Ulm, Germany \\ ${ }^{*}$ Corresponding author: J.Woods@soton.ac.uk
}

Received 18 December 2018; accepted 12 January 2019; posted 15 January 2019 (Doc. ID 354725); published 26 February 2019

\begin{abstract}
We investigate antireflection (AR) coating materials for two different operation lasing regimes requiring broad spectral bandwidth. We characterize high-power continuous-wave $(\mathrm{CW})$ wavelength-tunable vertical-externalcavity surface-emitting semiconductor lasers and their passive mode-locking capabilities when using semiconductor saturable absorber mirrors. One laser gain design was investigated with different single dielectric layers as AR coatings. The dielectric coating materials used were $\mathrm{SiO}_{2}, \mathrm{Al}_{2} \mathrm{O}_{3}, \mathrm{Ta}_{2} \mathrm{O}_{5}$, and $\mathrm{TiO}_{2}$. The $\mathrm{AR}$ coating was designed to reduce pump reflection and increase the confinement factor of the microcavity. Average power of $4.6 \mathrm{~W}$ in $\mathrm{CW}$ and a total wavelength tuning range of $42 \mathrm{~nm}$ has been observed with the $\mathrm{SiO}_{2}$-coated structure. The shortest pulse of $708 \mathrm{fs}$ was also observed for the $\mathrm{SiO}_{2}$-coated structure, with a corresponding $\mathrm{CW}$ wavelength tuning range of $36 \mathrm{~nm}$.
\end{abstract}

Published by The Optical Society under the terms of the Creative Commons Attribution 4.0 License. Further distribution of this work must maintain attribution to the author(s) and the published article's title, journal citation, and DOI.

https://doi.org/10.1364/JOSAB.36.000752

\section{INTRODUCTION}

The broad intrinsic gain bandwidth of optically pumped surface-emitting semiconductor laser chips is of interest for ultrashort pulse generation, as well as for wavelength-tunable narrowband emission. In passively mode-locked operation, vertical-external-cavity surface-emitting lasers (VECSELs) [1] incorporating semiconductor saturable absorber mirrors (SESAMs) [2] have achieved pulse durations down to $60 \mathrm{fs}$ in trains of pulses and around $100 \mathrm{fs}$ in single-pulse operation $[3,4]$ with an average power of $100 \mathrm{~mW}$. The disk laser geometry is also suitable for high-power operation, with a current record of more than $100 \mathrm{~W} \mathrm{CW}$ at $1 \mu \mathrm{m}$ emitted by a single optically pumped InGaAs/GaAs quantum well gain chip on a diamond submount [5]. SESAM mode-locking (ML) of structures of this type has allowed average power of up to $5.1 \mathrm{~W}[6]$, and peak power of up to $4.35 \mathrm{~kW}$ [7] to be achieved, but with longer pulse durations of $400 \mathrm{fs}$ or more. Wavelength-tunable operation of narrowband continuous-wave (CW) VECSEL has been demonstrated, [8-16]. Etalons have been primarily used in ML-VECSELs, and they are preferable as they can be thinner than birefringent filters (BRFs). Therefore, they do not introduce high dispersion in the cavity $[17,18]$. It is challenging to design gain chips that simultaneously fulfill the requirements for high power and broad gain bandwidth, whether in ultrashort pulse or tunable narrowband operation. Efficient pump absorption and high gain are achieved by using many quantum wells and a thick pump-absorbing barrier region in a resonant periodic gain configuration, with resonant enhancement. However, this design that leads to high gain also leads to narrowband filtering of the effective gain spectrum, which limits bandwidth and therefore tunability range. Different innovative schemes have been investigated to increase the tunability range of VECSELs, a two-chip gain cavity in Ref. [8], quantum well $(\mathrm{QW})$ thickness variation in the gain chip in Ref. [9], Fabry-Perot design with two resonant wavelengths [15], and parametric optimization of the layer thicknesses to produce a spectrally broadened confinement factor [16]. Another route to increasing tunability range involves membrane external cavity surface-emitting lasers (MECSELs) that do not have a distributed Bragg reflector (DBR), which results in a much-weakened microcavity effect [14]. Mode locking a laser that combines high output power and short pulses has similar challenges with developing a powerful tunable laser. The combination of the Watt-level average power and sub200 -fs pulse regime has not so far been achieved. At the heart of this challenge lie the above-mentioned characteristics of the gain spectrum of VECSEL structures, and several methods have been developed to characterize the gain spectrum [19-22]. 
Here we characterize laser performance both in the CW and mode-locked regime by altering the confinement factor of the microcavity by changing a single dielectric overlayer. This is a study of a simple yet flexible approach to the design of broadband gain chips, compatible with substrate removal for thermal management. A single dielectric overlayer is used to control coupling of pump light, penetration of the laser mode into the gain structure, and spectral filtering. Single-layer coatings of four different dielectric materials have been applied to thermally managed chips of the same InGaAs/GaAs quantum well gain structure. The refractive indices of the materials varied from 1.49 to 2.35. For each chip, we characterize both the tuning range in narrowband $\mathrm{CW}$ operation and the ultrashort pulse duration and spectrum in mode-locked operation. We achieve a $42 \mathrm{~nm}$ tuning range in $\mathrm{CW}$ wavelength tuning with 4.6 W average power and $708 \mathrm{fs}$ in the mode-locked regime with $1.3 \mathrm{~W}$ of output power. In both the $\mathrm{CW}$ and mode-locked regimes, we achieve broadband operation without sacrificing power. We show that the results in the two regimes are in agreement, and one simple architecture can be adapted for good performance in contrasted dynamical regimes.

\section{RESULTS}

The semiconductor gain structure was grown by a solidsource molecular beam epitaxy (MBE) on a (100)-oriented GaAs substrate. The structures consisted of a DBR, a resonant periodic gain region with seven InGaAs quantum wells, and the substrate. For the DBR, an alternating stack of $28 \mathrm{Al}_{0.15} \mathrm{Ga}_{0.85}$ As/AlAs pairs was chosen. With this material configuration and a Ti/Au metalization, the DBR provides high reflectivity for the emission wavelength $(>99.9 \%)$ and is near nonabsorbing for the pump wavelength $(808 \mathrm{~nm})$, providing resonant gain condition. For improved thermal management, the semiconductor structures were grown in reverse order, indium-soldered to a $300-\mu \mathrm{m}$-thick chemical vapor deposition (CVD) diamond heat spreader, and the substrate was subsequently removed by chemical wet etching.

The semiconductor structures were AR-coated for the pump wavelength to increase the pump absorption and overall efficiency. Four dielectric materials were used as coating material, $\mathrm{SiO}_{2}, \mathrm{Al}_{2} \mathrm{O}_{3}, \mathrm{Ta}_{2} \mathrm{O}_{5}$, and $\mathrm{TiO}_{2}$, with respective refractive indices of 1.49, 1.67, 2.07, and 2.35 and respective thicknesses of $135,122,96$, and $85 \mathrm{~nm}$. Further details for the semiconductor structure design and processing can be found in Ref. [10]. The respective calculated $\mid E$-field $\left.\right|^{2}$ intensities at the QWs for each AR-coated structure are shown in Fig. 1(a). The lower the refractive index of the AR coating, the more the design shifted from a resonant enhancement at the original design wavelength of $1040 \mathrm{~nm}$, down to an enhancement peak at shorter wavelengths down to $1020 \mathrm{~nm}$.

The laser cavity for the CW-tuning experiment was a straight cavity formed by the DBR and an output coupler $(\mathrm{OC})$, with a radius of curvature $(\mathrm{RoC})$ of $150 \mathrm{~mm}$ and a transmission of $1 \%$. The separation between the gain structure and the OC was $140 \mathrm{~mm}$. The gain chips were mounted on a

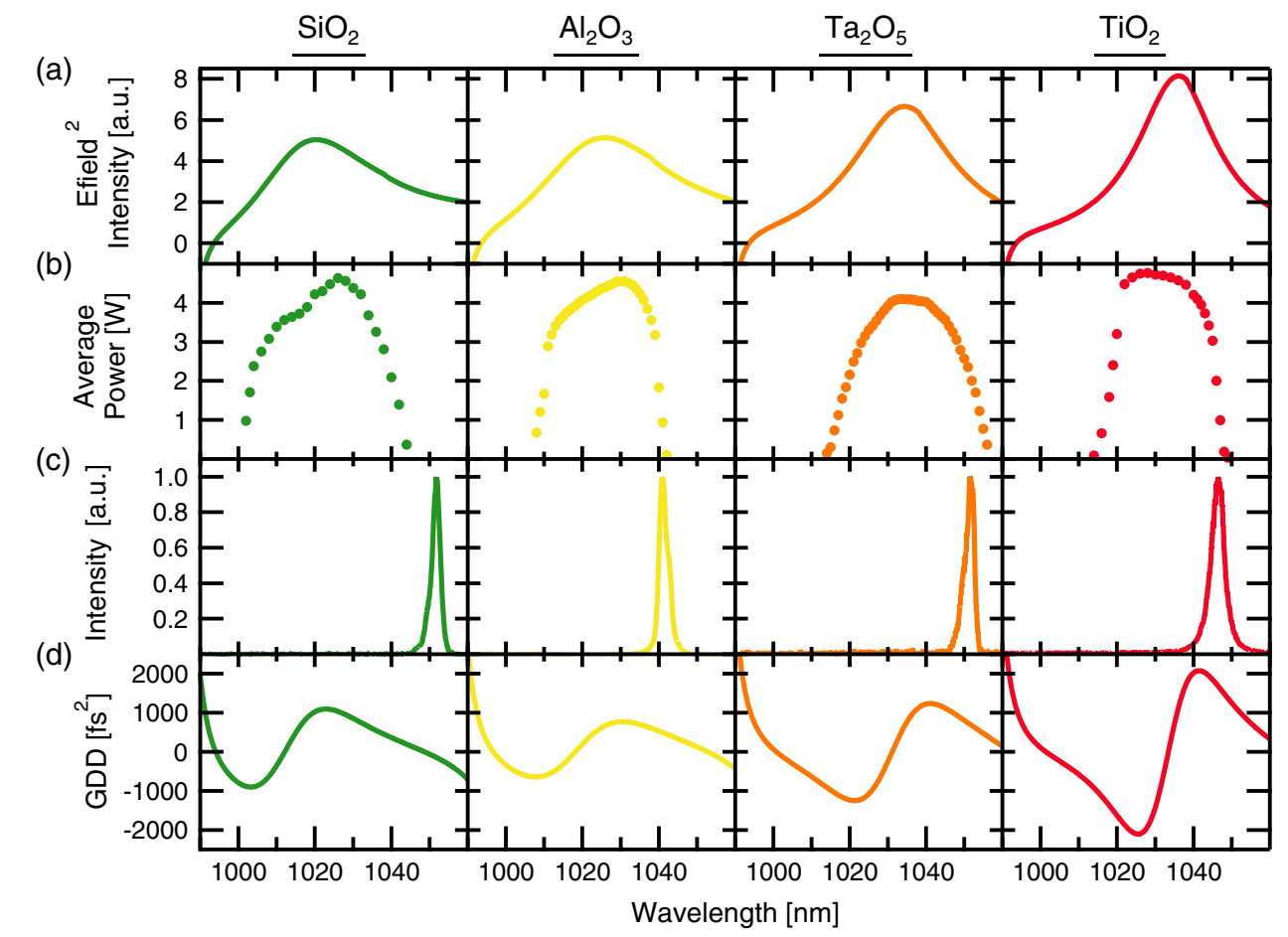

Fig. 1. (a) Respective calculated $\mid E$-field $\left.\right|^{2}$ intensities at the QWs for each AR-coated structure show the impact the refractive index of the coating layer has on the overall spectral enhancement profile of the gain structures. (b) Obtained average power during tuning of the emission wavelength via the 2-mm-thick BRF, for each dielectric coating. The incident pump power was $21.8 \mathrm{~W}$. (c) Corresponding lasing spectrum of each structure when mode-locked with the same SESAM and an incident pump power of $28.4 \mathrm{~W}$. (d) The calculated GDD profile for each of the AR-coated gain structures. 
Table 1. Summary of the CW Wavelength Tuning Results for Each Dielectric Coating Material with a Refractive Index of $n$, Where $\Delta \lambda$ Is the Total Tuning Range, $\Delta \lambda_{\text {FWHM }}$ Is the FWHM Tuning Range, and Max $\boldsymbol{P}_{\text {avg }}$ Is the Maximum Average Power Observed

\begin{tabular}{lcccc} 
Material & $\boldsymbol{n}$ & $\boldsymbol{\Delta} \boldsymbol{\lambda}(\mathbf{n m})$ & $\boldsymbol{\Delta} \boldsymbol{\lambda}_{\text {FWHM }}(\mathbf{n m})$ & $\boldsymbol{m a x} \boldsymbol{P}_{\text {avg }}(\mathbf{W})$ \\
\hline $\mathrm{SiO}_{2}$ & 1.49 & 42 & 36 & 4.6 \\
$\mathrm{Al}_{2} \mathrm{O}_{3}$ & 1.67 & 34 & 29 & 4.5 \\
$\mathrm{Ta}_{2} \mathrm{O}_{5}$ & 2.07 & 42 & 32 & 4.1 \\
$\mathrm{TiO}_{2}$ & 2.35 & 35 & 27 & 4.8
\end{tabular}

temperature-controlled heat sink, held at $18^{\circ} \mathrm{C}$, and were optically pumped at an angle of $23^{\circ}$ to the normal and with a pump spot size of $490 \times 470 \mu \mathrm{m}^{2}$ with $21.8 \mathrm{~W}$. The measured pump reflection was between $5-8 \%$. A 2-mm-thick BRF was placed inside the cavity at Brewster's angle to suppress the TE polarization. By rotating the BRF and without any other cavity adjustments, it was possible to tune the emission wavelength [8]. The resulting average output powers over the tuning range for each of the four different AR-coated gain structures are shown in Fig. 1(b) and summarized in Table 1.

The observed tuning range decreased with increasing refractive index of the AR coating material, with a full width at halfmaximum (FWHM) tuning range of $36 \mathrm{~nm}$ down to $27 \mathrm{~nm}$ for the lowest and highest refractive index material, respectively. This trend was expected from the $\mid$ E-field $\left.\right|^{2}$ calculations shown in Fig. 1(a). The only sample that did not follow this trend was the $\mathrm{Ta}_{2} \mathrm{O}_{5}$-coated sample, which had the second largest tuning range. Furthermore, it appears that the maximum output power also increased with decreasing refractive index, which is in contrast to the $\mid \mathrm{E}$-field $\left.\right|^{2}$ calculations, where one would expect higher maximum output powers from a higher refractive index material, which has a more resonant field enhancement. The |E-field $\left.\right|^{2}$ calculations, however, do not take into account the intrinsic QW gain spectrum. The $\mathrm{TiO}_{2}$-coated sample, with the highest refractive index, followed the expected trend and exhibited the highest peak power and lowest tuning bandwidth.

Following the wavelength tuning experiment, the samples have been investigated for their passively mode-locked performance. To passively mode lock the gain structures, a fast surfacerecombination SESAM was used. It consists of a DBR, a spacer layer, a single InGaAs-QW, and a $2 \mathrm{~nm}$ GaAs capping layer.

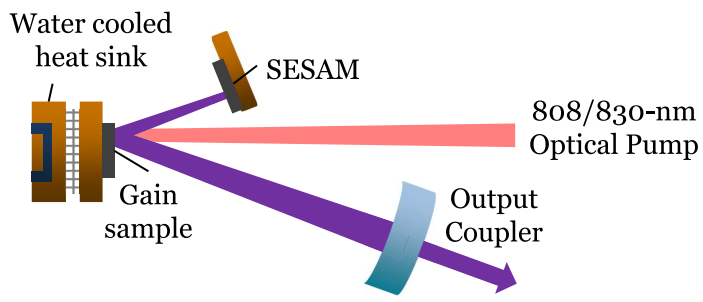

Fig. 2. VECSEL cavity for the passive mode-locking experiment consisting of three main components: the SESAM, the processed VECSEL gain chip (with a dielectric AR coating), and a $1.45 \%$ OC. The VECSEL is pumped with a $28.4 \mathrm{~W}, 808 \mathrm{~nm}$ fiber-coupled diode laser.
A detailed description of surface-recombination SESAMs for passive ML with VECSELs can be found in Ref. [1], and the specific SESAM design is described and used in Ref. [7].

The cavity geometry used for the ML experiment was a V-cavity, as shown in Fig. 2, formed by an OC with $100 \mathrm{~mm} \mathrm{RoC}$ and $1.45 \%$ transmission, the optically pumped gain structure, and the SESAM. The distance between the OC and the gain structure, and the gain and the SESAM, were $68 \mathrm{~mm}$ and $31 \mathrm{~mm}$, respectively. The pump laser delivered up to $28.4 \mathrm{~W}$ with an emission wavelength of $808 \mathrm{~nm}$, and the pump spot radius on the gain sample was $180 \mu \mathrm{m}$. Both the gain sample and SESAM were mounted on Peltier temperature-controlled water-cooled copper heat sinks, where the temperatures of the heat sinks were kept at $15^{\circ} \mathrm{C}$ and $-20^{\circ} \mathrm{C}$, for the gain sample and SESAM, respectively. The cooling of the SESAM to $-20^{\circ} \mathrm{C}$ was necessary to increase the obtainable average power.

All four samples exhibited stable ML; the spectra are shown in Fig. 1(c), and the autocorrelations in Fig. 3. The pulse durations and average powers are summarized in Table 2. The sample coated with $\mathrm{SiO}_{2}$ exhibited the shortest pulse duration of $708 \mathrm{fs}$, and the observed pulse durations increased up to 1.63 ps for the $\mathrm{TiO}_{2}$-coated sample, following the expected trend with increasing refractive index. If we compare these results to the CW tuning results in Table 1, it can be seen that pulse durations achieved for the samples with a broader FWHM tuning range tend to generate shorter pulse durations. However, none of the pulses were transform-limited, ranging from 1.9 to 6.8 times the transform limit, increasing with the refractive index. We have so far not accounted for the group delay dispersion (GDD), which can have a significant

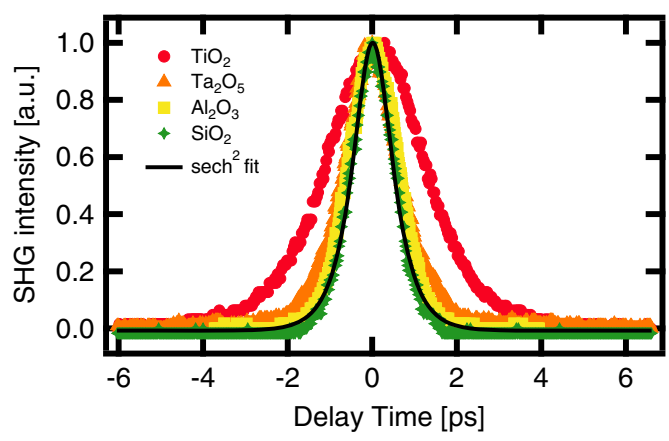

Fig. 3. Autocorrelations obtained for each AR-coated structure. The solid line is a sech ${ }^{2}$ fit for the $\mathrm{SiO}_{2}$-coated sample, with a pulse duration of $708 \mathrm{fs}$.

Table 2. Observed Pulse Duration, $\tau_{\text {pulse; }}$; Average Power, $\boldsymbol{P}_{\text {avg; }}$; and Transform Limit Product during Mode Locking for Each Dielectric AR Coating

\begin{tabular}{lcccc}
\hline Material & $\boldsymbol{n}$ & $\boldsymbol{\tau}_{\text {pulse }}(\mathbf{f s})$ & $\boldsymbol{P}_{\text {avg }}(\mathbf{m W})$ & Transform Limit \\
\hline $\mathrm{SiO}_{2}$ & 1.49 & 708 & 1300 & 1.9 \\
$\mathrm{Al}_{2} \mathrm{O}_{3}$ & 1.67 & 820 & 720 & 2.1 \\
$\mathrm{Ta}_{2} \mathrm{O}_{5}$ & 2.07 & 983 & 460 & 5.1 \\
$\mathrm{TiO}_{2}$ & 2.35 & 1630 & 460 & 6.8
\end{tabular}


impact on the chirp of the generated pulse [23]. The GDD calculations for the four different structures are shown in Fig. 1(d), where it can be seen that with increasing the refractive index of the AR coating, the GDD increases too. The high positive GDD of almost $2000 \mathrm{fs}^{2}$ for the $\mathrm{TiO}_{2}$-coated sample is likely to cause the high time-bandwidth product. The GDD of the SESAM is calculated to be close to zero and becomes comparatively negligible as the pulse will pass twice through the gain and only once through the SESAM.

The average power during ML ranged from 1300 to $460 \mathrm{~mW}$ for the four structures where the mode-locked average power was higher for the structures with a lower refractive index of the AR coating. This is again opposite to what one would expect from the $\mid \mathrm{E}$-field $\left.\right|^{2}$ calculations. However, the observed trend is consistent with the measured maximum average powers of the CW tuning experiment, but significantly more pronounced. In the work of Mangold et al. [21], a $25 \mathrm{~nm}$ and $30 \mathrm{~nm}$ FWHM of the unsaturated gain spectrum gave $780 \mathrm{fs}$ and $625 \mathrm{fs}$ pulse durations, respectively. In comparison, the $36 \mathrm{~nm}$ FWHM CW tuning bandwidth structure that we achieve here with the silica coating should produce shorter pulses, if dispersion compensation is used.

\section{CONCLUSIONS}

In summary, we presented CW tuning ranges at the multiwatt average power level with a total tuning range of up to $42 \mathrm{~nm}$ with the $\mathrm{SiO}_{2}$-coated structure. Furthermore, we have investigated high-efficiency high-power broadly wavelengthtunable AR-coated gain structures and their suitability for passive ML performance. Broader CW tuning bandwidths were corresponding to shorter pulse durations; the shortest pulse generated was $708 \mathrm{fs}$ with $1.3 \mathrm{~W}$ average power with the $\mathrm{SiO}_{2}$-coated structure, which also exhibited the broadest tuning width. However, all pulses had a transform limit of $>1.9$; thus, shorter pulse durations of broadly wavelengthtunable AR-coated gain structures should be possible with correct dispersion management. We show that $\mathrm{CW}$ wavelength tuning performance could be used as a predictor of performance for mode-locked operation, which may further help to drive sample designs towards the watt-level, sub-200-fs pulse duration regime.

Funding. Engineering and Physical Sciences Research Council (EPSRC) (EP/M013294/1).

Acknowledgment. All data supporting this study are openly available from the University of Southampton repository [24].

\section{REFERENCES}

1. A. Garnache, S. Hoogland, A. C. Tropper, I. Sagnes, G. Saint-Girons, and J. S. Roberts, "Sub-500-fs soliton-like pulse in a passively modelocked broadband surface-emitting laser with $100 \mathrm{~mW}$ average power," Appl. Phys. Lett. 80, 3892-3894 (2002).

2. U. Keller, K. J. Weingarten, F. X. Kärtner, D. Kopf, B. Braun, I. D. Jung, R. Fluck, C. Hönninger, N. Matuschek, and J. Aus der Au, "Semiconductor saturable absorber mirrors (SESAM's) for femtosecond to nanosecond pulse generation in solid-state lasers," IEEE J. Sel. Top. Quantum Electron. 2, 435-453 (1996).

3. A. H. Quarterman, K. G. Wilcox, V. Apostolopoulos, Z. Mihoubi, S. P. Elsmere, I. Farrer, D. A. Ritchie, and A. Tropper, "A passively modelocked external-cavity semiconductor laser emitting 60-fs pulses," Nat. Photonics 3, 729-731 (2009).

4. D. Waldburger, S. M. Link, M. Mangold, C. G. E. Alfieri, E. Gini, M. Golling, B. W. Tilma, and U. Keller, "High-power 100 fs semiconductor disk lasers," Optica 3, 844-852 (2016).

5. B. Heinen, T. L. Wang, M. Sparenberg, A. Weber, B. Kunert, J. Hader, S. W. Koch, J. V. Moloney, M. Koch, and W. Stolz, "106 W continuous-wave output power from vertical-external-cavity surfaceemitting laser," Electron. Lett. 48, 516-517 (2012).

6. M. Scheller, T. L. Wang, B. Kunert, W. Stolz, S. W. Koch, and J. V. Moloney, "Passively modelocked VECSEL emitting 682 fs pulses with $5.1 \mathrm{~W}$ of average output power," Electron. Lett. 48, 588-589 (2012).

7. K. G. Wilcox, A. C. Tropper, H. E. Beere, D. A. Ritchie, B. Kunert, B. Heinen, and W. Stolz, "4.35 kW peak power femtosecond pulse mode-locked VECSEL for supercontinuum generation," Opt. Express 21, 1599-1605 (2013).

8. L. Fan, M. Fallahi, A. R. Zakharian, J. Hader, J. V. Moloney, R. Bedford, J. T. Murray, W. Stolz, and S. W. Koch, "Extended tunability in a two-chip VECSEL," IEEE Photon. Technol. Lett. 19, 544-546 (2007).

9. J. Paajaste, S. Suomalainen, R. Koskinen, A. Harkonen, M. Guina, and M. Pessa, "High-power and broadly tunable GaSb-based optically pumped VECSELs emitting near $2 \mu \mathrm{m}$," J. Cryst. Growth 311, 1917-1919 (2009).

10. A. Hein, S. Menzel, and P. Unger, "High-power high-efficiency optically pumped semiconductor disk lasers in the green spectral region with a broad tuning range," Appl. Phys. Lett. 101, 111109 (2012).

11. M. Butkus, J. Rautiainen, O. G. Okhotnikov, C. J. Hamilton, G. G. Malcolm, S. S. Mikhrin, I. L. Krestnikov, D. D. Livshits, and E. U. Rafailov, "Quantum dot based semiconductor disk lasers for 1-1.3 $\mu \mathrm{m}$," IEEE J. Sel. Top. Quantum Electron. 17, 1763-1771 (2011).

12. D. A. Nakdali, M. K. Shakfa, M. Gaafar, M. Butkus, K. A. Fedorova, M. Zulonas, M. Wichmann, F. Zhang, B. Heinen, A. Rahimi-Iman, W. Stolz, E. U. Rafailov, and M. Koch, "High-power quantum-dot verticalexternal-cavity surface-emitting laser exceeding 8 W," IEEE Photon. Technol. Lett. 26, 1561-1564 (2014).

13. V. J. Wittwer, C. A. Zaugg, Z. Sun, D. Popa, S. Milana, T. S. Kulmala, R. S. Sundaram, M. Mangold, M. Golling, Y. Lee, J. H. Ahn, U. Keller, and A. C. Ferrari, "Graphene saturable absorbers for VECSELs," Proc. SPIE 8966, 89660X (2014).

14. Z. Yang, A. R. Albrecht, J. G. Cederberg, and M. Sheik-Bahae, "80 nm tunable DBR-free semiconductor disk laser," Appl. Phys. Lett. 109, 022101 (2016).

15. A. Broda, A. Wójcik-Jedlińska, I. Sankowska, M. Wasiak, M Wieckowska, and J. Muszalski, "A 95-nm-wide tunable two-mode vertical external cavity surface-emitting laser," IEEE Photon. Technol. Lett. 29, 2215-2218 (2017).

16. C. Borgentun, J. Bengtsson, A. Larsson, F. Demaria, A. Hein, and $P$. Unger, "Optimization of a broadband gain element for a widely tunable high-power semiconductor disk laser," IEEE Photon. Technol. Lett. 22, 978-980 (2010).

17. O. J. Morris, K. G. Wilcox, C. R. Head, A. P. Turnbull, P. J. Mosley, A. H. Quarterman, H. J. Kbashi, I. Farrer, H. E. Beere, D. A. Ritchie, and A. C. Tropper, "A wavelength tunable 2-ps pulse VECSEL," Proc. SPIE 8242, 824212 (2012).

18. C. A. Zaugg, Z. Sun, V. J. Wittwer, D. Popa, S. Milana, T. S. Kulmala, R. S. Sundaram, M. Mangold, O. D. Sieber, M. Golling, Y. Lee, J. H. Ahn, A. C. Ferrari, and U. Keller, "Ultrafast and widely tuneable vertical-external-cavity surface-emitting laser, mode-locked by a graphene-integrated distributed Bragg reflector," Opt. Express 21, 31548-31559 (2013).

19. M. E. Barnes, Z. Mihoubi, K. G. Wilcox, A. H. Quarterman, I. Farrer, D. A. Ritchie, A. Garnache, S. Hoogland, V. Apostolopoulos, and A. C. Tropper, "Gain bandwidth characterization of surface-emitting 
quantum well laser gain structures for femtosecond operation," Opt. Express 18, 21330-21341 (2010).

20. C. Borgentun, J. Bengtsson, and A. Larsson, "Direct measurement of the spectral reflectance of OP-SDL gain elements under optical pumping," Opt. Express 19, 16890-16897 (2011).

21. M. Mangold, V. J. Wittwer, O. D. Sieber, M. Hoffmann, I. L. Krestnikov, D. A. Livshits, M. Golling, T. Südmeyer, and U. Keller, "VECSEL gain characterization," Opt. Express 20, 4136-4148 (2012)
22. C. R. Head, K. G. Wilcox, A. P. Turnbull, O. J. Morris, E. A. Shaw, and A. C. Tropper, "Saturated gain spectrum of VECSELs determined by transient measurement of lasing onset," Opt. Express 22, 6919-6924 (2014).

23. M. Hoffmann, O. D. Sieber, D. J. H. C. Maas, V. J. Wittwer, M. Golling, T. Südmeyer, and U. Keller, "Experimental verification of soliton-like pulse-shaping mechanisms in passively mode-locked VECSELs," Opt. Express 18, 10143-10153 (2010).

24. https://doi.org/10.5258/SOTON/D0742. 